\section{Integrative Medicine \\ International}

Integr Med Int 2014;1:93-101

\title{
Clinical Study on Chronic Pain in Parkinson's Disease Patients in Shanghai, China
}

\author{
Weidong Pan ${ }^{a}$ Jun Liud Quidong Wang ${ }^{\mathrm{e}}$ Hua Lu ${ }^{\mathrm{c}}$ Yu Bai ${ }^{f} \quad \mathrm{Yi} \mathrm{Liu}^{\mathrm{g}}$ \\ Xiangjun Chen ${ }^{\text {h }}$ Wentao Lig Wenwei Li ${ }^{i}$ Yu Song ${ }^{\text {a }}$ Heng Wang ${ }^{j}$ \\ Yuncheng $\mathrm{Wu}^{\mathrm{k}}$ Meng $\mathrm{Xu}^{\mathrm{a}}$ Hua Zhoub \\ Departments of a Neurology and ${ }^{b}$ Cardiology, and ${ }^{c}$ Center for Clinical Effectiveness \\ Evaluation, Shuguang Hospital Affiliated to Shanghai University of Traditional Chinese \\ Medicine, ${ }^{\mathrm{d}}$ Department of Neurology, Ruijin Hospital Affiliated to Shanghai Jiaotong \\ University, ${ }^{e}$ Department of Neurology, Pudong New Area Hospital of Traditional Chinese \\ Medicine, ${ }^{f}$ Department of Neurology, Putuo District Center Hospital Affiliated to Shanghai \\ University of Traditional Chinese Medicine, 9 Department of Neurology, Shanghai Hospital \\ of Traditional Chinese Medicine, hepartment of Neurology, Huashan Hospital Affiliated to \\ Fudan University, 'Laboratory for Neurology, Institute of Integrative Medicine, Zhongshan \\ Hospital Affiliated to Fudan University, ${ }^{j}$ Department of Internal Medicine of Traditional \\ Chinese Medicine, Chongming Branch Hospital Affiliated to Shanghai Jiaotong University, \\ and kDepartment of Neurology, Shanghai First People's Hospital, Shanghai Jiaotong \\ University School of Medicine, Shanghai, China
}

\section{Key Words}

Pain in Parkinson's disease - Questionnaires · Management of pain · Asymmetric pain . Integrative medicine

\begin{abstract}
Background/Aims: Pain in Parkinson's disease (PD) may be more distressing than that in other nonmotor disabilities. The aims of the present study were to assess the prevalence and identify the epidemiological characteristics of PD-related pain among patients with idiopathic PD in Shanghai, China. Methods: A total of 1,058 patients with definite idiopathic PD were investigated using a structure questionnaire in 12 hospitals in Shanghai. The severity of their motor disorders, antiparkinson treatments, and pain-related observations, such as time points for the onset of pain, duration and degree, body localization, external influences, and treatments for pain, were collected for analysis. Results: Approximately $28 \%$ (296 subjects) of all PD patients suffered from PD-related pain. Female patients with pain had a higher age level, were older at PD onset, had a higher frequency of pain, a higher frequency of pain before the
\end{abstract}

Weidong Pan, Jun Liu, and Qiudong Wang contributed equally as first authors. 
onset of PD symptoms, a longer duration of pain, and a higher incidence of pain in the early stage compared to male patients. Larger levodopa-equivalent doses, higher frequencies of sleep disorders and/or motor fluctuation, and/or dyskinesia were found in patients with pain compared to patients without pain. The most frequent pain type was musculoskeletal pain followed by dystonia. The male group had a much shorter pain duration than the female group. The patients experienced more pain before taking antiparkinson drugs compared to after treatment. Conclusion: Chronic PD-related pain is a frequent complaint and complex in PD. The challenge of managing pain in PD patients will hopefully someday result in specific and effective treatment strategies.

(C) 2014 S. Karger AG, Base

\section{Introduction}

The pain symptoms associated with Parkinson's disease (PD) account for substantial morbidity in up to $80 \%$ of the patients [1]. There is abundant indirect evidence indicating that abnormal somatosensory processing in the basal ganglia might involve the substantia nigra, caudate, putamen, globus pallidus, thalamus, and their interconnections [2]. Musculoskeletal pain (due to PD rigidity/skeletal deformity), radicular neuropathic pain (due to root lesion, focal or peripheral neuropathy), dystonic pain (often due to the effects of medications), and central or primary pain (considered a disease-specific symptom in PD) have been categorized for PD patients $[1,3]$. Sometimes patients may also report akathisia (a feeling of restlessness). It might be described as vague, poorly located, discomfort, tension, tickling, and burning, and it may precede the motor symptoms of PD, with little response to dopaminergic therapy. Most research on pain in PD has been conducted in Western countries [4, 5], and little is known about the prevalence and associated factors of pain among PD patients in China. Therefore, we analyzed the data of PD patients in China to identify the epidemiological characteristics of pain among Chinese patients living in Shanghai because the constitutions and medical conditions of Chinese PD patients are very different from those of individuals of other ethnic groups [6]. The objectives of this prospective study were to establish the frequency of pain among these patients, and to identify clinical characteristics and pharmacological therapies that could be related to pain manifestations.

\section{Materials and Methods}

\section{Subjects}

Data were collected by interviewing 1,058 patients with definite idiopathic PD diagnosed according to the UK Brain Bank criteria [7], who were surveyed at 12 hospitals, including 7 Western medicine hospitals and 5 integrative medicine hospitals, in Shanghai over an 8-month period (between July 1, 2013 and February 28 2014). A comprehensive clinical protocol was followed to collect a large dataset of clinical features for each patient as described below. The patients' age ranged between 35 and 87 years. The Shuguang Hospital Affiliated to Shanghai University of Traditional Chinese Medicine approved the protocol, and all participants provided informed consent.

Survey Tool

After signing an informed consent form, the enrolled PD patients were asked to fill out a structured questionnaire (original questionnaire printed in Chinese) that included two parts. One part was on demographic information, the pathogenic features of PD, and previous and current use of antiparkinson treatments. The Hoehn and Yahr (H\&Y) score and the Unified Parkinson's Disease Rating Scale (UPDRS) were assessed as being appropriate to indicate stage and severity of PD. Patients were excluded if they had parkinsonism due to other causes or if they were unable to give consent. The other part of the question- 
naire contained pain-related observations, such as data regarding time points for the onset of pain (wake time or sleep time, before or after PD diagnosis, before or after taking antiparkinson medicine), duration and degree, body localization, external influences, and pain treatments. Chronic pain was defined as the occurrence of pain related to PD and associated with fluctuations of the motor symptoms, cramps, or other pain sensations not explained by coexisting physical or mental problems, and was classified into five types as musculoskeletal, radicular neuropathic, dystonia, central or primary, and unspecified. Patients had suffered from pain for 3 days or more per week for at least 3 months prior to inclusion in the study. The Visual Analog Scale (VAS) score marks the maximal pain at a score of 10 ( $\leq 5$ is defined as mild, $>5$ is defined as severe) and shows that the duration of pain was used to assess the symptoms of pain [8]. Patients with dementia, clinical or electrophysiological evidence of peripheral neuropathy, or a disease that might be associated with pain such as diabetes mellitus, coexistence of arthrosis, tension headache, or neck ache not associated with PD were excluded. Severe fluctuations in PD, concurrent existence of epilepsy, active malignancy, polyneuropathy, or other serious diseases of somatic or psychiatric origin that could interfere with the study were also exclusion criteria. The patients were further asked about or tested for nonmotor symptoms such as sleep disorders, depression, postural hypotension, anosphrasia, cognitive disorders, hypohidrosis, urine retention, sexual dysfunction, and restless legs syndrome. Sleep disorders were assessed using the Parkinson's Disease Sleep Scale (SPSS) [9], and depression was assessed with the Hamilton Depression Scale (HAM-D) [10]. If motor problems impeded this activity, a family member or caregiver was allowed to help with filling out the questionnaire forms in the presence of the physician and interviewers. Twelve specially trained investigators performed all assessments as interviewers. One investigator conducted all interviews.

\section{Statistical Methods}

SPSS (version 19.0, SPSS China Inc.) was used for statistical analysis. All data are expressed as means \pm SD. The rate or constituent ratio was calculated for categorical variables. In order to investigate the clinical differences between males and females, we assessed all parameters in terms of not only the total patient population, but also for male and female patients with PD. The t test, $\chi^{2}$ test, or Wilcoxon rank-sum test was used to compare or analyze the constituent ratio between the categorical variables with respect to proportions (presence of different symptoms or characteristics). A significant difference was defined as $\mathrm{p}<0.05$.

\section{Results}

The questionnaires were completed in more than $97 \%$ (1,029 patients) of the cases by PD patients. The basic characteristics of the PD patients are presented in tables 1 and 2 . The mean age was $59.7 \pm 8.8$ years (range $35-87$ ) and the mean PD duration was $5.9 \pm 7.8$ years (range 0.8-22) for all patients who completed the questionnaire. The mean educational duration was $8.2 \pm 4.5$ years for all patients, and the number of education years was significantly longer in males than females ( $p<0.05$, t test). Approximately 28\% (296 subjects) of all PD patients suffered from PD-related pain, and the frequency of pain was the same for males and females. The survey revealed a shorter duration of pain symptoms in males compared to females ( $p<0.05$, t test), and the mean duration of pain was approximately $5.7 \pm 5.5$ years for all patients with pain. Almost all pain patients (238 cases, $80.4 \%$ of all pain patients) suffered pain after PD onset compared to before PD onset ( $p<0.001$, t test; table 1$)$. We also found smaller numbers of male patients with pain compared to females in the mild stage (H\&Y score $1-2.5, \mathrm{p}<0.05$, Wilcoxon rank-sum test; table 2). No such relations were found for age at PD onset, duration of PD, and UPDRS scores among pain patients, male and female patients without pain, and the total population.

Table 3 indicates the clinical treatments and characteristics of patients with PD. It shows significantly larger levodopa-equivalent doses in the pain patients compared to patients without pain in the total population, while both male groups (male patients with and without pain) showed significantly larger levodopa-equivalent doses than females $(p<0.05, t$ test $)$. 
Pan et al.: Clinical Study on Chronic Pain in Parkinson's Disease Patients in Shanghai, China

Table 1. Basic characteristics of all PD patients $(n=1,029)$

\begin{tabular}{llcr}
\hline \multirow{2}{*}{ Characteristic } & \multicolumn{2}{c}{ Patients with PD } & \multirow{2}{*}{ Total } \\
\cline { 2 - 3 } & female & male & \\
\hline Age, years & $63.8 \pm 9.6$ & $55.3 \pm 11.3^{*}$ & $59.7 \pm 8.8$ \\
Education level, years & $7.8 \pm 4.1$ & $9.3 \pm 4.9^{*}$ & $8.2 \pm 4.5$ \\
Age at PD onset, years & $58.5 \pm 6.1$ & $52.7 \pm 7.3^{*}$ & $56.1 \pm 5.6$ \\
Time since PD diagnosis, years & $5.1 \pm 6.3$ & $6.8 \pm 6.7^{* *}$ & $5.9 \pm 7.8$ \\
Frequency of PD with pain & $38.7(155 / 401)$ & $22.5(141 / 628)^{* *}$ & $28.8(296 / 1,029)$ \\
PD with pain & & & 19.9 \\
$\quad$ Before onset & 29.3 & $11.6^{* *}$ & $80.4^{* * *}$ \\
$\quad$ After onset & 70.7 & $88.4^{*}$ & $5.7 \pm 5.5$ \\
Duration of pain, years & $6.8 \pm 7.1$ & $4.1 \pm 3.9^{* *}$ & \\
\hline
\end{tabular}

Values are means \pm SD or percentages, and values in parentheses are numbers. ${ }^{*} \mathrm{p}<0.05,{ }^{* *} \mathrm{p}<0.01$ compared with females, ${ }^{* * *} \mathrm{p}<0.001$ compared with pain before PD onset.

${ }^{a}$ The localizations or pain symptoms are the same as after PD onset for the PD patients with pain. ${ }^{b}$ Values are given as means for PD with pain.

Table 2. Basic characteristics of all PD patients $(n=1,029)$

Table 3. Clinical treatment and characteristics of patients with $\mathrm{PD}(\mathrm{n}=1,029)$

\begin{tabular}{lll}
\hline Sex & \multicolumn{2}{l}{ H\&Y score, $\%$} \\
\cline { 2 - 3 } & $1-2.5$ & $>2.5$ \\
\hline Female & $47.6^{*}$ & 52.4 \\
Male & $36.3^{*}$ & 63.7 \\
Total & $41.9^{*}$ & 57.9 \\
\hline
\end{tabular}

${ }^{*} \mathrm{p}<0.05$, compared with the H\&Y score $>2.5$, t test.

\begin{tabular}{|c|c|c|}
\hline Characteristic & $\begin{array}{l}\text { PD with pain } \\
(n=296)\end{array}$ & $\begin{array}{l}\text { PD without pain } \\
(n=733)\end{array}$ \\
\hline With sleep disorder & $76.7^{* *}$ & 43.6 \\
\hline With motor fluctuation & $68.6^{* *}$ & 39.7 \\
\hline With dyskinesias & $84.5^{* *}$ & 44.1 \\
\hline H\&Y score $1-2.5$ & $41.9^{*}$ & 54.3 \\
\hline H\&Y score $>2.5$ & $51.1^{*}$ & 45.7 \\
\hline UPDRS total score & $37.2 \pm 8.7$ & $36.8 \pm 9.2$ \\
\hline UPDRS score part III & $24.8 \pm 8.9$ & $23.9 \pm 7.7$ \\
\hline HAM-D & $15.9 \pm 4.7$ & $14.1 \pm 7.3$ \\
\hline L-dopa, mg.day ${ }^{-1}$ & $532.5 \pm 139.9^{*}$ & $489.5 \pm 181.6$ \\
\hline DRD2, mg. day ${ }^{-1}$ & $0.73 \pm 0.49^{*}$ & $0.67 \pm 0.51$ \\
\hline
\end{tabular}

Values are means \pm SD or percentages. L-dopa = Levodopa/ decarboxylase inhibitor; DRD2 = dopamine receptor agonist 2. * p < $0.05,{ }^{* *} \mathrm{p}<0.01$, compared with PD without pain, t test. 
Table 4. Characteristics of localization, time points, types, duration and drug correlation of pain in PD patients with pain

\begin{tabular}{|c|c|c|c|c|c|c|}
\hline \multirow[t]{2}{*}{ Characteristic } & & \multicolumn{2}{|c|}{ PD with pain $(n=296)$} & \multirow[t]{2}{*}{ Total } & \multirow[t]{2}{*}{$\chi^{2}$ value } & \multirow[t]{2}{*}{$\mathrm{p}$ value } \\
\hline & & female & male & & & \\
\hline \multirow{4}{*}{ Localization } & left limbs & 38.6 & 27.9 & 33.3 & \multirow[t]{4}{*}{3.857} & \multirow[t]{4}{*}{0.027} \\
\hline & right limbs & 41.3 & 49.7 & 45.5 & & \\
\hline & trunk & 11.5 & 10.8 & 11.2 & & \\
\hline & migratory & 8.6 & 11.6 & 10.1 & & \\
\hline \multirow[t]{4}{*}{ Time points } & awake time & 59.8 & 63.5 & 61.7 & \multirow[t]{4}{*}{12.358} & \multirow[t]{4}{*}{0.0018} \\
\hline & sleep time & 27.4 & 24.9 & 26.2 & & \\
\hline & all day or variable & & & & & \\
\hline & interval & 12.8 & 11.6 & 12.2 & & \\
\hline \multirow{5}{*}{$\begin{array}{l}\text { Frequency of every type } \\
\text { of pain }\end{array}$} & musculoskeletal & 51.3 & 38.4 & 45.0 & \multirow[t]{5}{*}{13.711} & \multirow[t]{5}{*}{0.0013} \\
\hline & radicular neuropathic & 10.9 & 12.3 & 11.6 & & \\
\hline & dystonia & 18.2 & 15.4 & 16.8 & & \\
\hline & central or primary & 8.4 & 7.9 & 8.2 & & \\
\hline & unspecified & 11.2 & 25.7 & 18.5 & & \\
\hline Duration of pain, h. day ${ }^{-1}$ & & $16.3 \pm 4.4$ & $11.2 \pm 6.9^{*}$ & & $t=12.9$ & 0.027 \\
\hline \multirow{3}{*}{ Drug correlation $^{\mathrm{a}}$} & before & 57.2 & 61.3 & 59.3 & \multirow[t]{3}{*}{12.972} & \multirow[t]{3}{*}{0.0011} \\
\hline & after & 23.4 & 17.6 & 20.5 & & \\
\hline & no & 19.4 & 21.1 & 20.3 & & \\
\hline \multirow[t]{2}{*}{ VAS score } & $\leq 5$ & 37.8 & 59.3 & 48.6 & \multirow[t]{2}{*}{$Z=-3.630$} & \multirow[t]{2}{*}{0.067} \\
\hline & $>5$ & 62.2 & 40.7 & 51.5 & & \\
\hline
\end{tabular}

Values are percentages or means \pm SD. $* p<0.05$, compared with females.

${ }^{a}$ Correlation with taking oral antiparkinson medications.

Higher frequencies in sleep disorders, and/or motor fluctuation, and/or dyskinesia were observed in patients with pain compared to patients without pain ( $\mathrm{p}<0.01, \mathrm{t}$ test) in males, females, and the total population, respectively. No significant differences in sleep disorders, motor fluctuation, depression, postural hypotension, anosphrasia, cognitive disorders, hypohidrosis, urine retention, sexual dysfunction, and restless legs syndrome were observed among males, females, and the total population in the no pain group. The only exception was for patients without dyskinesia; here, the number of females was larger than the number of males ( $\mathrm{p}<0.05$, t test; table 3$)$.

Table 4 shows the relationship of the categorical variables of the patients with PD-related pain. It displays a significantly smaller number of left limb localization of pain in males compared to females. The most frequent pain localization was in the arms and legs $(p=0.027$, $\chi^{2}$ test), while the most frequent pain time points were during the time awake in all pain patients $\left(p=0.0018, \chi^{2}\right.$ test). The most frequent pain type was musculoskeletal followed by dystonia in all pain patients. The mean pain duration was $16.3 \pm 4.4,11.2 \pm 6.9$, and $13.8 \pm$ $5.6 \mathrm{~h}$ per day for females, males, and the total population, respectively. The pain duration was much shorter in the male group than in the female group ( $p<0.05, t$ test). More patients reported that the pain appeared before taking antiparkinson drugs ( $p=0.0011, \chi^{2}$ test). Females experienced more severe pain (VAS score $>5$ ) than males, but there was no significant difference between the severity of pain (VAS score $\leq 5$ or VAS score $>5$ ) in the total population ( $\mathrm{p}=0.067$, Wilcoxon rank-sum test). 


\section{Discussion}

In this study, we have analyzed different aspects of chronic PD-related pain, focusing on the patients' own experiences, and observed that asymmetric pain is a common complication (28.7\% of the total population) in patients with PD. Pain in PD is not visible and is therefore difficult for physicians to immediately identify, and patients often do not mention that they are experiencing a lot of pain. Often, even if a patient complains of pain, the physician does not respond to or prioritize it. These are the reasons for the inadequate management of pain in PD patients. Pain in PD is reported to be much less frequent in general PD studies, with the exception of special pain-related studies in PD [4]. In our study, the morbidity of pain in PD patients was much lower than that in other studies $[11,12]$. In addition to the above reasons, it might also have led to many integrative therapy (IT) treatments such as complementary and alternative therapy in the Shanghai population. Furthermore, it has been reported that these therapies have potent effects on motor and nonmotor disorders in patients with PD [13-15]. Our study also revealed gender differences in pain in PD patients living in Shanghai. Female patients with pain had a higher age, were older at PD onset, had a higher frequency of pain, a higher frequency of pain before the onset of PD symptoms, a longer duration of pain, and a higher incidence of pain in the early stages (H\&Y score 1-2.5) compared to male patients (table 1). Pathologically, patients with PD are characterized by loss of dopaminergic neurons in the substantia nigra and presence of Lewy bodies in the remaining neurons. Braak and Del Tredici [16] reported that PD starts in the dorsal motor nucleus of the medulla oblongata, and gradually the disease process ascends to the brain stem to involve the substantia nigra and eventually the cerebral cortex. Due to its widespread involvement in PD, many nonmotor symptoms are known and many of these symptoms, such as pain, may be observed before the motor disturbance (table 1).

Reduced endogenous pain inhibition is presumed to be the mechanism underlying the common presence of pain in patients with PD [17]. This controversy might be related to the potential effect of dopamine on pain inhibition pathways. Since at least one of the cortically based descending pain inhibitory pathways is partially dopaminergic (rostral agranular insular cortex) [18], the dopaminergic denervation indicates a higher level of pain and suggests that the causes of pain might be related to movement disorders as well as the loss of dopaminergic neurons in the substantia nigra [18] and arise from central basal ganglia dysfunction [5, 19]. Evidence has shown an increased incidence of nondystonic or musculoskeletal pain and pain relief experienced by the patient subsequent to levodopa therapy as well as an increased pain threshold observed during the on-period [19]. Patients with sleep disorders, and/or with motor fluctuation, and/or with dyskinesia will suffer PD-related pain more easily and need a higher dosage of levodopa compared to patients without pain (table 3 ). This may be explained by the fact that the causes of pain in PD patients might be correlated with the severity of the neuron degeneration [2], and furthermore, PD patients with pain have a significantly lower threshold for heat-produced pain stimuli than pain-free PD patients and healthy controls [4]. A major finding of the study was that significant differences with respect to gender, geographic distribution, and age (tables 1,4) were similar to those reported by Marcus et al. [20], who found that pain, fatigue, and psychiatric complaints were more commonly reported in women. Reduced pain thresholds and tolerance $[5,19,21]$ and/or increased responses to pain stimuli were observed in females [22]. Schestatsky et al. [19] and Brefel-Courbon et al. [11] discovered that during off-periods, patients with PD and central pain had a lower threshold for pain caused by thermal stimuli. Furthermore, positron emission tomography studies of pain-free PD patients during the off-period revealed increased activation caused by pain stimuli of the right insular and prefrontal cortex and in the left cingulate cortex, compared to results from control subjects. Patients with motor fluctuation and more 
severe patients (H\&Y score $>2.5$ ) seem to suffer pain to a greater extent than the overall population of patients with pain (tables 1,3 ).

Differential activation of the right and left hemispheres in response to somatosensory stimuli has been described in health [23] and in chronic pain [24, 25]. A previous study reported that selective grey matter atrophy was observed in the right nucleus accumbens as well as in the right insula and right ventromedial prefrontal cortex in chronic regional pain syndrome patients, regardless of the affected body side [26]. More right limb pain was found in our study from Shanghai, and therefore, there might be different pathological reasons that cause the laterality of pain in PD patients (table 4).

In early stages, pain may result from a stiff shoulder, rigidity or dystonia. In advanced stages, pain may be caused by motor fluctuations (dyskinesia or off-period dystonia), and may be radicular or due to musculoskeletal problems [27, 28]. In most of the published work, pain in PD was found to be related to a musculoskeletal cause or dystonia [1, 3, 29-32]. Musculoskeletal pain (44.4\%) and dystonia-related pain $(19.1 \%)$ were the most common types of pain (table 4). Musculoskeletal problems are common in patients with PD, and in some studies, appear to be related to the presence of rigidity and akinesia [30]. One of the most common musculoskeletal problems in PD is shoulder stiffness, and a stiff shoulder may be the first sign of PD. Dystonia may occur as an early manifestation of dopaminergic deficiency or may be related to levodopa therapy. Central pain in PD, unrelated to motor symptoms or their consequences, is suggested to be a direct effect of the disease itself [33]. Central parkinsonian pain is complex and may involve sensory pathways within the basal ganglia and the thalamocortical-basal ganglia circuits [34]. In this study, $11.1 \%$ of the patients were deemed to have PD-related central pain.

Therefore, the management of pain in PD patients should seek to alleviate the increased dystonic and central pain by optimizing dopaminergic therapy. Pain associated with dystonia may be related to involuntary muscular contraction and may be best managed with options like botulinum toxin, whereas neuropathic pain may be related to a central dopaminergic deficit and may benefit from therapeutic options targeted at this pathology [32]. In addition, anti-inflammatory medication may help reduce local irritation and opiates may help modulate pain pathways [35]. Once pain in PD patients has been correctly classified according to subtype and separated from pain associated with comorbid conditions (most commonly osteoarthritis but also other conditions such as diabetes and rheumatological conditions), the management of pain with various pharmacological and nonpharmacological therapies may be initiated. Patients who have pain associated with rigidity due to parkinsonism may have damage to nonneural tissue due to muscle/joint stretching and subsequent inflammation [36]. These patients may benefit from nonpharmacological treatment such as stretching exercises, repetitive transcranial magnetic stimulation or cranial electrotherapy stimulation. Repetitive transcranial magnetic stimulation therapy has been shown to be effective in the management of dystonic pain [35]. Dystonic pain may also present as frozen shoulder or as foot dystonia, and analgesia in these presentations may include botulinum toxin injection in addition to optimizing dopaminergic therapy with levodopa or other agents [35, 37]. IT might be an effective treatment for pain [38,39] or other nonmotor disorders in PD [13-15]. Our previous study has demonstrated that more than $68 \%$ of the patients with PD have used IT including TCM, acupuncture, massage and Tai Chi Quan in Shanghai, and more than half of them could draw benefits from IT in treating their nonmotor disorders [6]. We did not collect information on the IT treatments or evaluate its effects in our study, and this is a deficiency of our study on pain in PD.

However, we did not find such a correlation, consistent with the results of Tinazzi et al. [30], who reported that pain was not associated with self-reported depression. Nonetheless, the relatively small sample size in our study does not provide sufficient power to assess all 
potential correlations between the presence of pain and other demographic or phenotypical parameters, or concomitant medication. Another limitation is the absence of studies about the correlation between IT treatments and pain morbidity; thus, it is not possible to conclude with certainty whether pain in a PD population is more frequent than in a demographically matched PD group treated with IT [40].

\section{Conclusion}

Asymmetric pain is a common complication in patients with PD, and our study has confirmed that chronic PD-related pain is complex in nature. The pain may be correlated with the PD onset, age, gender, levodopa-equivalent doses, motor fluctuation, and the severity of PD. The challenge of managing pain in PD patients requires experience and judgment of the clinician. An improved understanding of pain in PD will hopefully someday result in specific and effective treatment strategies. Future studies should address pain-reducing therapies, including integrative medicines.

\section{Disclosure Statement}

The authors have no stock ownership in medically related fields. The authors declare that they have no conflicts of interest regarding the publication of this paper.

\section{References}

1 Beiske AG, Loge JH, Ronningen A, Svensson E: Pain in Parkinson's disease: prevalence and characteristics. Pain 2009;141:173-177.

-2 Granovsky Y, Schlesinger I, Fadel S, Erikh I, Sprecher E, Yarnitsky D: Asymmetric pain processing in Parkinson's disease. Eur J Neurol 2013;20:1375-1382.

3 Goetz CG, Tanner CM, Levy M, Wilson RS, Garron DC: Pain in Parkinson's disease. Mov Disord 1986;1:45-49.

4 Djaldetti R, Shifrin A, Rogowski Z, Sprecher E, Melamed E, Yarnitsky D: Quantitative measurement of pain sensation in patients with Parkinson disease. Neurology 2004;62:2171-2175.

5 Zambito Marsala S, Tinazzi M, Vitaliani R, Recchia S, Fabris F, Marchini C, Fiaschi A, Moretto G, Giometto B, Macerollo A, et al: Spontaneous pain, pain threshold, and pain tolerance in Parkinson's disease. J Neurol 2011; 258:627-633.

6 Lu H, Pan W, Wang J, Wu C, Gong F, et al: The current status of integrative therapies in treating Parkinson's disease in six general hospitals in Shanghai. Int J Integr Med 2013;1:17.

7 Gibb WR, Lees AJ: The relevance of the Lewy body to the pathogenesis of idiopathic Parkinson's disease. J Neurol Neurosurg Psychiatry 1988;51:745-752.

-8 Latham J: Assessment and measurement of pain. Eur J Cancer Care (Engl) 1994;3:75-78.

-9 Chaudhuri KR, Pal S, DiMarco A, Whately-Smith C, Bridgman K, Mathew R, Pezzela FR, Forbes A, Hogl B, Trenkwalder C: The Parkinson's disease sleep scale: a new instrument for assessing sleep and nocturnal disability in Parkinson's disease. J Neurol Neurosurg Psychiatry 2002;73:629-635.

10 Williams JB: A structured interview guide for the Hamilton Depression Rating Scale. Arch Gen Psychiatry 1988;45:742-747.

11 Brefel-Courbon C, Payoux P, Thalamas C, Ory F, Quelven I, Chollet F, Montastruc JL, Rascol O: Effect of levodopa on pain threshold in Parkinson's disease: a clinical and positron emission tomography study. Mov Disord 2005;20:1557-1563.

12 Rana AQ, Kabir A, Jesudasan M, Siddiqui I, Khondker S: Pain in Parkinson's disease: analysis and literature review. Clin Neurol Neurosurg 2013;115:2313-2317.

13 Pan W, Kwak S, Liu Y, Sun Y, Fang Z, Qin B, Yamamoto Y: Traditional Chinese medicine improves activities of daily living in Parkinson's disease. Parkinsons Dis 2011;2011:789506.

14 Pan W, Liu Y, Fang Z, Zhu X, Pan W, Kwak S, Yamamoto Y: A compound belonging to traditional Chinese medicine improves nocturnal activity in Parkinson's disease. Sleep Med 2011;12:307-308.

15 Pan W, Kwak S, Li G, Chen Y, Cai D: Therapeutic effect of Yang-Xue-Qing-Nao granules on sleep dysfunction in Parkinson's disease. Chin Med 2013;8:14. 
Braak H, Del Tredici K: Invited Article: Nervous system pathology in sporadic Parkinson disease. Neurology 2008;70:1916-1925.

17 Burkey AR, Carstens E, Jasmin L: Dopamine reuptake inhibition in the rostral agranular insular cortex produces antinociception. J Neurosci 1999;19:4169-4179.

18 Ortega-Legaspi JM, de Gortari P, Garduno-Gutierrez R, Amaya MI, Leon-Olea M, Coffeen U, Pellicer F: Expression of the dopaminergic D1 and D2 receptors in the anterior cingulate cortex in a model of neuropathic pain. Mol Pain 2011;7:97.

19 Schestatsky P, Kumru H, Valls-Sole J, Valldeoriola F, Marti MJ, Tolosa E, Chaves ML: Neurophysiologic study of central pain in patients with Parkinson disease. Neurology 2007;69:2162-2169.

-20 Marcus SM, Kerber KB, Rush AJ, Wisniewski SR, Nierenberg A, Balasubramani GK, Ritz L, Kornstein S, Young EA, Trivedi MH: Sex differences in depression symptoms in treatment-seeking adults: confirmatory analyses from the Sequenced Treatment Alternatives to Relieve Depression study. Compr Psychiatry 2008;49:238246.

21 Horn ME, Alappattu MJ, Gay CW, Bishop M: Fear of severe pain mediates sex differences in pain sensitivity responses to thermal stimuli. Pain Res Treat 2014;2014:897953.

-22 Tinazzi M, Recchia S, Simonetto S, Defazio G, Tamburin S, Moretto G, Fiaschi A, Miliucci R, Valeriani M: Hyperalgesia and laser evoked potentials alterations in hemiparkinson: evidence for an abnormal nociceptive processing. J Neurol Sci 2009;276:153-158.

23 Coghill RC, Gilron I, Iadarola MJ: Hemispheric lateralization of somatosensory processing. J Neurophysiol 2001;85:2602-2612.

24 Hsieh JC, Belfrage M, Stone-Elander S, Hansson P, Ingvar M: Central representation of chronic ongoing neuropathic pain studied by positron emission tomography. Pain 1995;63:225-236.

-25 Hsieh JC, Hannerz J, Ingvar M: Right-lateralised central processing for pain of nitroglycerin-induced cluster headache. Pain 1996;67:59-68.

26 Geha PY, Baliki MN, Harden RN, Bauer WR, Parrish TB, Apkarian AV: The brain in chronic CRPS pain: abnormal gray-white matter interactions in emotional and autonomic regions. Neuron 2008;60:570-581.

27 Ford B: Pain in Parkinson's disease. Clin Neurosci 1998;5:63-72.

28 Sage JI: Pain in Parkinson's disease. Curr Treat Options Neurol 2004;6:191-200.

-29 Lee MA, Walker RW, Hildreth TJ, Prentice WM: A survey of pain in idiopathic Parkinson's disease. J Pain Symptom Manage 2006;32:462-469.

30 Tinazzi M, Del Vesco C, Fincati E, Ottaviani S, Smania N, Moretto G, Fiaschi A, Martino D, Defazio G: Pain and motor complications in Parkinson's disease. J Neurol Neurosurg Psychiatry 2006;77:822-825.

-31 Defazio G, Berardelli A, Fabbrini G, Martino D, Fincati E, Fiaschi A, Moretto G, Abbruzzese G, Marchese R, Bonuccelli $U$, et al: Pain as a nonmotor symptom of Parkinson disease: evidence from a case-control study. Arch Neurol 2008;65:1191-1194.

-32 Negre-Pages L, Regragui W, Bouhassira D, Grandjean H, Rascol O; DoPaMiP Study Group: Chronic pain in Parkinson's disease: the cross-sectional French DoPaMiP survey. Mov Disord 2008;23:1361-1369.

-33 Canavero S: Central pain and Parkinson disease. Arch Neurol 2009;66:282-283; author reply 283 Chudler EH, Dong WK: The role of the basal ganglia in nociception and pain. Pain 1995;60:3-38. Sophie M, Ford B: Management of pain in Parkinson's disease. CNS Drugs 2012;26:937-948. Perez-Lloret S, Rey MV, Dellapina E, Pellaprat J, Brefel-Courbon C, Rascol O: Emerging analgesic drugs for Parkinson's disease. Expert Opin Emerg Drugs 2012;17:157-171.

37 Ha AD, Jankovic J: Pain in Parkinson's disease. Mov Disord 2012;27:485-491.

38 Lee C, Crawford C, Teo L, Spevak C; Active Self-Care Therapies for Pain (PACT) Working Group: An analysis of the various chronic pain conditions captured in a systematic review of active self-care complementary and integrative medicine therapies for the management of chronic pain symptoms. Pain Med 2014;15(suppl 1):S96-S103.

-39 Crawford C, Lee C, Buckenmaier C 3rd, Schoomaker E, Petri R, Jonas W; Active Self-Care Therapies for Pain (PACT) Working Group: The current state of the science for active self-care complementary and integrative medicine therapies in the management of chronic pain symptoms: lessons learned, directions for the future. Pain Med 2014;15(suppl 1):S104-S113.

40 Pan W, Zhou H: Inclusion of integrative medicine in clinical practice. Integr Med Int 2014;1:1-4. 